\title{
Computational Study of the Interactions between Antimalarial Chemotherapies with Folate Pathway Receptors and Telomerase Reverse Transcriptase
}

\author{
Djogang Lucie Karelle1,2, Forlemu Neville3 ${ }^{3}$ Emadak Alphonse ${ }^{1 *}$, Njabon Njankwa Eric1, \\ Issofa Patouossa1, Nenwa Justin² \\ ${ }^{1}$ Physical and Theoretical Chemistry Laboratory, Faculty of Science, University of Yaounde I, Yaounde, Cameroon \\ ${ }^{2}$ Applied Inorganic Chemistry Laboratory, Faculty of Science, University of Yaounde I, Yaounde, Cameroon \\ ${ }^{3}$ School of Science \& Technology (Chemistry), Georgia Gwinnett College, Lawrenceville, USA \\ Email: *emadak@yahoo.com
}

How to cite this paper: Karelle, D.L., Neville, F., Alphonse, E., Eric, N.N., Patouossa, I. and Justin, N. (2021) Computational Study of the Interactions between Antimalarial Chemotherapies with Folate Pathway Receptors and Telomerase Reverse Transcriptase. Computational Chemistry, 9, 197-214.

https://doi.org/10.4236/cc.2021.93011

Received: May 13, 2021

Accepted: July 23, 2021

Published: July 26, 2021

Copyright $\odot 2021$ by author(s) and Scientific Research Publishing Inc. This work is licensed under the Creative Commons Attribution International License (CC BY 4.0).

http://creativecommons.org/licenses/by/4.0/

\section{(c) (i) Open Access}

\begin{abstract}
Malaria is a life-threatening disease responsible for half a million death annually, and with nearly half of the world's population at risk. The rapid drop in observed cases of malaria in the last two decades has been due to a combination of preventive and therapeutic remedies. However, the absence of a vaccine, new antimalarial chemotherapies and increased parasitic resistance have led to a plateau of infections and renewed research interest in target human and Plasmodium (the malaria parasite) receptors and new drugs. In this study, the impact of mutation on the affinity on antimalarial drugs with the bifunctional enzyme complex, dihydrofolate reductase (DHFR) is explored. In addition, homology modeling is used to build the three-dimensional models of the enzymes Plasmodium telomerase reverse-transcriptase ( $p f-T E R T)$ and Plasmodium dihydropteroate synthetase ( $p f-D H P S)$ to determine their affinity with antimalarial drugs. The interaction energies and stable complexes formed between these enzymes and antimalarial drugs (chloroquine, artemisinin, primaquine, pyrimethamine, sulfadoxine and pentamidine) were modelled using AutoDock vina. Our data indicate that $p f-T E R T$ and $p f-D H P S$ form stable complexes with the antimalarial ligands with affinity ranging from -4.0 to $-6.9 \mathrm{kcal} / \mathrm{mol}$. The affinity with crystal structures of DHFR receptors was higher ranging from -6.0 to $-10.0 \mathrm{kcal} / \mathrm{mol}$. The affinity to DHFR also decreases with the mutation a confirmation of the source of resistance. The highest affinity interaction for all the receptors modeled is observed with Artemisinin a benchmark antimalarial drug. This can be attributed to the size, shape and dipolar surface of the ligand. The observed com-
\end{abstract}


plexes are stabilized by strategic active site polar and non-polar contacts.

\section{Keywords}

Malaria, Binding Energy, Dihydrofolate Reductase, Dihydropteroate Synthetase, Free Energy, Telomerase Reverse-Transcriptase, Plasmodium falciparum, Antimalarial Drugs

\section{Introduction}

Malaria is one of the debilitating parasitic diseases caused by the protozoan parasite Plasmodium falciparum [1] [2]. It affects a large part of the world's population and causes an average of half a million deaths each year [3]. The folate metabolic pathway is a critical source of metabolites and intermediates necessary for the synthesis of nucleotides and amino acids, paramount to the survival of the Plasmodium parasite [4] [5]. This pathway has been targeted for both treatment and prophylaxis of the disease [6]. Two key enzymes involved in this pathway, namely dihydrofolate reductase (DHFR) and dihydropteroate synthetase (DHPS) are highlighted in supplemental materials (Scheme 1).

Dihydrofolate reductase (DHFR) catalyzes the reduction of dihydrofolate to tetrahydrofolate a key precursor in the synthesis of nucleotide bases (pyrimidines, and thymidylic acid) and amino acids, required by bacteria, Plasmodium as well as normal and cancerous human cells. Several antifolate drugs have been shown to prevent replication of parasitic DNA and therefore one effective way to fight malaria. This is primarily achieved by inhibiting DHFR enzymatic activity thereby blocking cell division of the parasite at the schizont stage [7] [8]. There is, however, evidence suggesting that the activity of antifolate drugs like chloroquine and pyrimethamine has been undermined by several cases of parasitic resistance caused by DHFR mutations [9]. The mutations affect the polarity and size of the DHFR binding domains. It is therefore vital to understand how such subtle changes affect the molecular details of the interactions. Dihydropteroate synthase (DHPS) is the other folate pathway enzyme that catalyzes the conversion of $p$-aminobenzoic acid to dihydrofolic acid. Targeting this enzyme is also crucial because it is only expressed in parasitic cells and therefore a good target for drug specificity. In addition to folate pathway enzymes, Plasmodium reverse transcriptase telomerase ( $p f-T E R T$ ) was recently identified as a target receptor for the development of antimalarial chemotherapies was discussed in a recent study by Parida and collaborators, Plasmodium falciparum telomerase reverse transcriptase ( $p f-T E R T)$ also emerged as a plausible target receptor for antimalarial activity and his sequence provides valuable information for the design of specific anti-telomerase drugs [10].

In order to understand how these enzymes interact with antimalarial drugs at the molecular level requires the availability of good three-dimensional structures of the target receptors. The three-dimensional structures of some of these target 
receptors (DHFR) have been solved and available in the protein databank [11]. The absence of X-ray crystal structures for $p f-D H P S$ and $p f-T E R T$ receptors pose a challenge.

In this study, homology modeling and molecular docking are employed, to characterize the interactions between key enzyme receptors in the critical pathways described above with current antimalarial medications. Specifically: 1) Homology modeling is used to build three-dimensional structures of Plasmodium enzymes (DHPS and TERT); 2) AutoDock Vina is used to estimating the binding affinity of antimalarial medications (chloroquine, pyrimethamine, sulfadoxine, pentamidine, primaquine and artemisinin) with human and parasitic receptors.

The main objective is to determine whether DHPS and $p f-T E R T$ can also serve as potential target receptors for antimalarial activity. In addition, we explore the difference in affinity between DHFR mutants and antimalarial drugs. The affinity and interaction patterns for multiple target enzyme receptors in different pathways can help explain the wide range of action of many antimalarial medications. Autodock Vina is used to rapidly access the stability and rank the drug-receptor complexes, with the goal to determine and explain any observed specificity and or selectivity of current antimalarial drugs like pyrimethamine. The drugs with enhanced selectivity will serve as pharmacophores for the development of novel sulfonamides, pyrimethamine or chloroquine-based derivatives.

\section{Molecular Systems and Computational Methods}

\subsection{Molecular Systems}

The interactions between 6 antimalarial drugs used in and target receptors from multiple metabolic pathways were characterized using molecular docking. The docked complexes were generated using a combination of the Chimera molecular visualization package, and docking software AutoDock Vina [12]. The threedimensional structures of the antimalarial drugs (artemisinin, pyrimethamine, primaquine, chloroquine, pentamidine, and sulfadoxine) were downloaded from DrugBank database and represented in (Figure 1) [13].

\subsection{Homology Modeling of Target Protein Receptors}

Homology modeling is a computational technique used to approximate the tertiary structure of a protein whose primary amino acid sequence is available, based on the coordinates known 3D homologous structures. The atomic coordinates of the target protein is obtained based from the homologue structure based on the similarity scores obtained from the sequence and structural alignments. The four steps implemented to build the structures for $p f-D H P S$ and $p f-T E R T$ include: target identifications, sequence alignment, model building and refinement [14] [15]. In this study, the blast sequence tool in Uniprot database was used to perform a sequence alignment between the target sequences ( $p f$-TERT-uniprot ID 23479392 and $p f$-DHPS-uniprot ID Q5PZ24) and numerous template sequences 


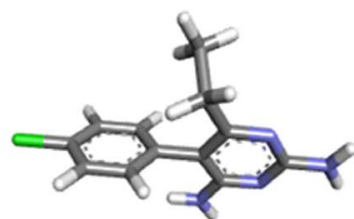

(A)

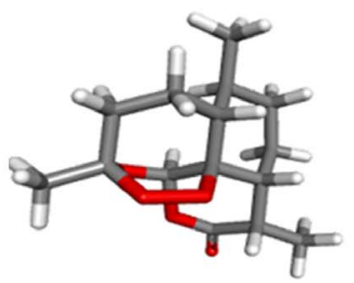

(D)

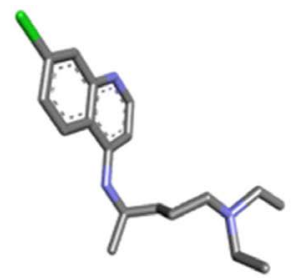

(B)

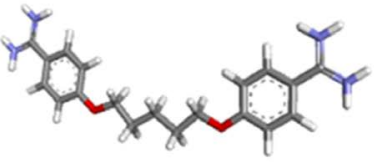

(E)

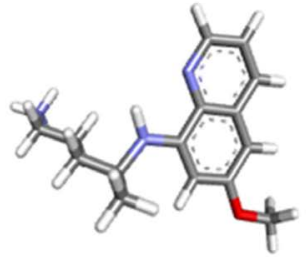

(C)

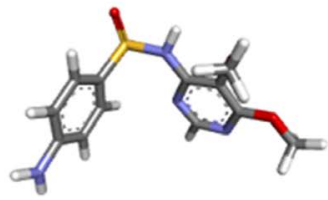

(F)

Figure 1. Chemical Structures of antimalarial ligands. (A) Pyrimethamine; (B) Chloroquine; (C) Primaquine; (D) Artemisinin; (E) Pentamidine and (F) Sulfadoxine.

Table SM1 [16] [17]. The crystal structure of Plasmodium falciparum Glutathione reductase, accession code (1ONF) was used to build pf-TERT model, while Plasmodium falciparum hydroxymethyldihydropterin pyrophosphokinase-dihydropteroate synthase accession code (6KCL) was used to build $p f-D H P S)$. The models were built using Modeller with the Chimera graphics user interface [18]. The atomic overlaps in the resulting models were relaxed using the Chimera energy minimization tool. In each case, the AMBER force field was used with a dielectric constant of 78.3 (water). A strategy of steepest descents followed by conjugate gradients was adopted for a total of 10,000 iterations [19].

\subsection{Docking Simulations}

Two representative receptors from the folate pathway were used in these simulations. These include dihydrofolate reductase thymidylate synthase (DHFR-TS), and dihydropteroate synthase (DHPS). DHFR-TS mutations have been linked to resistance to antimalarial activity. The three-dimensional structures of DHFR have been solved and were downloaded from the RCSB Protein Data Bank are represented in (Figure 2). These include; human DHFR (h-DHFR and pdb-ID-3GYF), wild type Plasmodium DHFR (wt-pf-DHFR and pdb-ID-4DPD) double mutant Plasmodium ( $d$-pf-DHFR and pdb-ID-3UM6) and quadruple mutant Plasmodium DHFR (q-DHFR and pdb-ID-4DP3) [20] [21] [22].

The target receptor enzymes with solved X-ray crystallized three-dimensional structures were refined prior to docking using Chimera. This involved adding deleted atoms (polar hydrogens) from crystal structures, removing all non-standard ligands and relaxing the enzyme structures. Other receptors used in the docking simulations include the homology models of $p f$-TERT and $p f-D H P S$ (Figure 3).

The USCF Chimera Dock Prep tool was used to refine the receptor molecules and Gasteiger charges. A global search of the best bound complexes, ranked and 


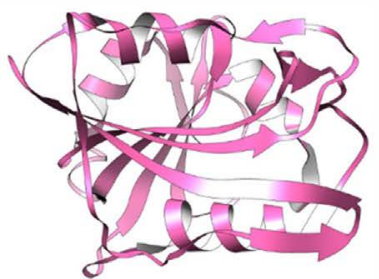

(A)

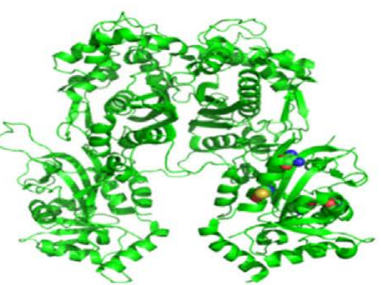

(B)

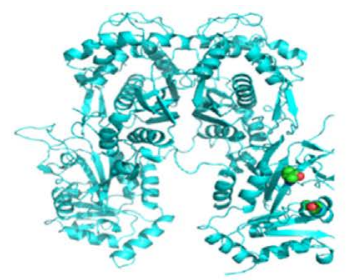

(C)

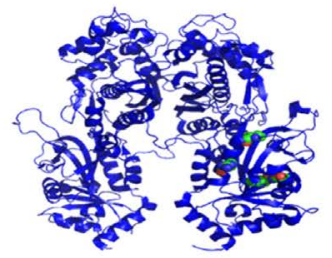

(D)

Figure 2. (A) Crystal Structure of human DHFR; (B) Wild type $p f$-DHFR, double mutant $p f$-DHFR with mutations A16V and S108N highlighted; and quadruple mutant $p f$-DHFR with mutations N51I, C59R, S108N, and I164L highlighted.

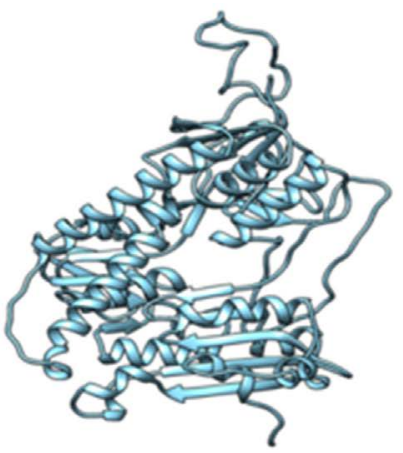

(A)

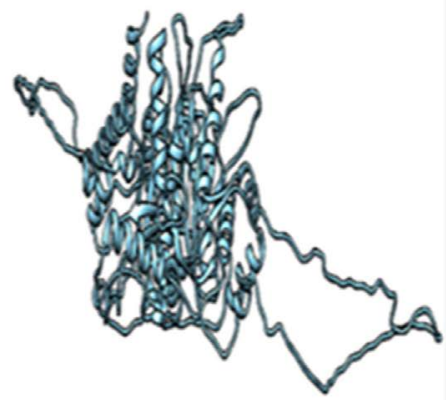

(C)

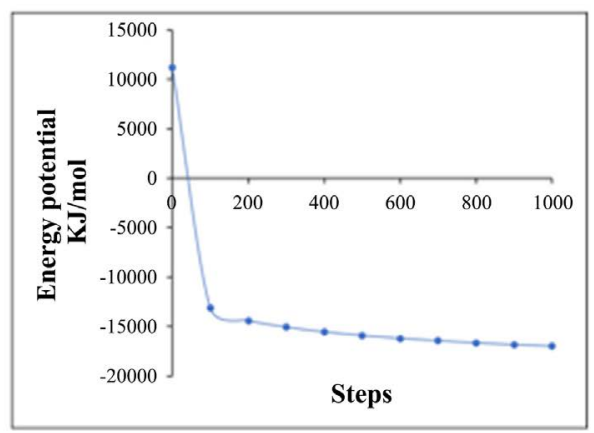

(B)

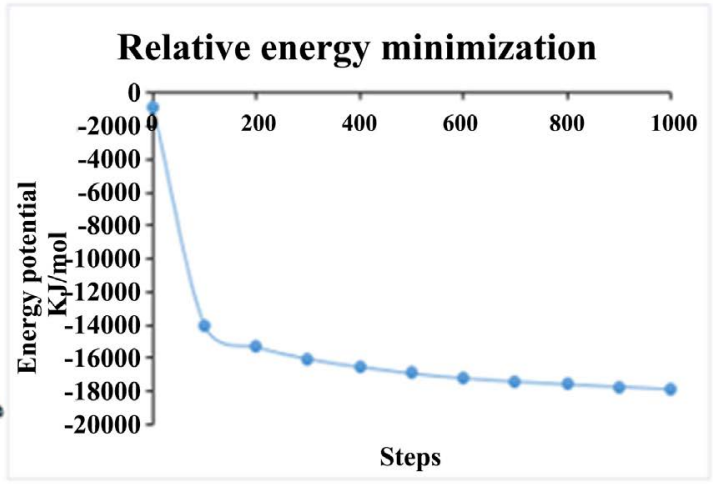

(D)

Figure 3. (A) Homology model of Plasmodium Teleromase; (B) Energy minimization profile; (C) homology model of plasmodium falciparum DHPS; and (D) Energy minimization profile.

scored based on favorable hydrophobic, hydrogen bond and van der Waals interactions was performed using the AutoDock Vina software [23]. Docking was performed using the protein and ligand coordinates along with docking grid box with size $50 \AA \times 50 \AA \times 50 \AA$ center around active site of DHFR [24]. The docked complex with lowest binding energy is then extracted for further analysis.

\section{Results and Discussion}

\subsection{Relative Binding Energies}

The docking simulations screened the affinity and interactions of six current an- 
timalarial medications with a multiple target receptors to understand what is driving these interactions at a molecular level, determine the key residues involved in binding and whether some receptor mutations significantly affect the binding process. The AutoDock Vina binding energies between the antimalarial drugs and folate pathway enzyme DHFR are represented in Figure 4. We also explored the interactions of $p f-T E R T$ and $p f-D H P S$ using three dimensional structures obtained from homology modeling. All the antimalarial drugs modeled (pyrimethamine, primaquine, pentamidine, chloroquine, artemisinin and sulfadoxine) formed complexes with Plasmodium enzyme receptors.

The folate acid pathway enzymes were targeted because of their role in the synthesis of nucleotides and amino acids, critical for the survival of the plasmodium parasite. There are some indications that antimalarial drugs can interfere with the telomerase activities to induce shortening of telomere and chromosome loss [25]. This can be effective in fighting the malaria scourge caused by plasmodium parasite [26]. All ligands were successfully docked to the target DHFR enzyme receptors. Artemisinin, the benchmark drug present in many effective combination therapies against malaria interact more favorably with all the DHFR enzymes. There is some evidence indicating that the mutations in DHFR negatively impact the affinity of interactions involving artemisinin (Figure 4(A)). For example, the affinity becomes less negative from h-DHFR and $w t$-DHFR to quadruple mutant $p f$-DHFR ( $q m$-pf-DHFR) (Figure $4(\mathrm{~A})$ ). The trend is slightly reversed for the interactions between pyrimethamine and the Plasmodium enzymes. For example, there is a bigger difference in energy between h-DHFR, or $w t-p f$-DHFR and $d m$ - $p f$-DHFR which are respectively of $-1.5 \mathrm{kcal} / \mathrm{mol}$ and -1.1 $\mathrm{kcal} / \mathrm{mol}$ contrary to $q m-p f$-DHFR whose difference is only $-0.4 \mathrm{kcal} / \mathrm{mol}$ with h-DHFR. The results of (Figure $4(\mathrm{~A})$ ) are different from those obtained by Forlemu and coworkers [5]. This difference could be explained not only by the active site chosen to perform the docking calculation but above all the nature of the amino acids (polar and non-polar) which involved these sites. The antimalarial drugs were also docked to the $p f$-DHPS and $p f$-TERT models built using

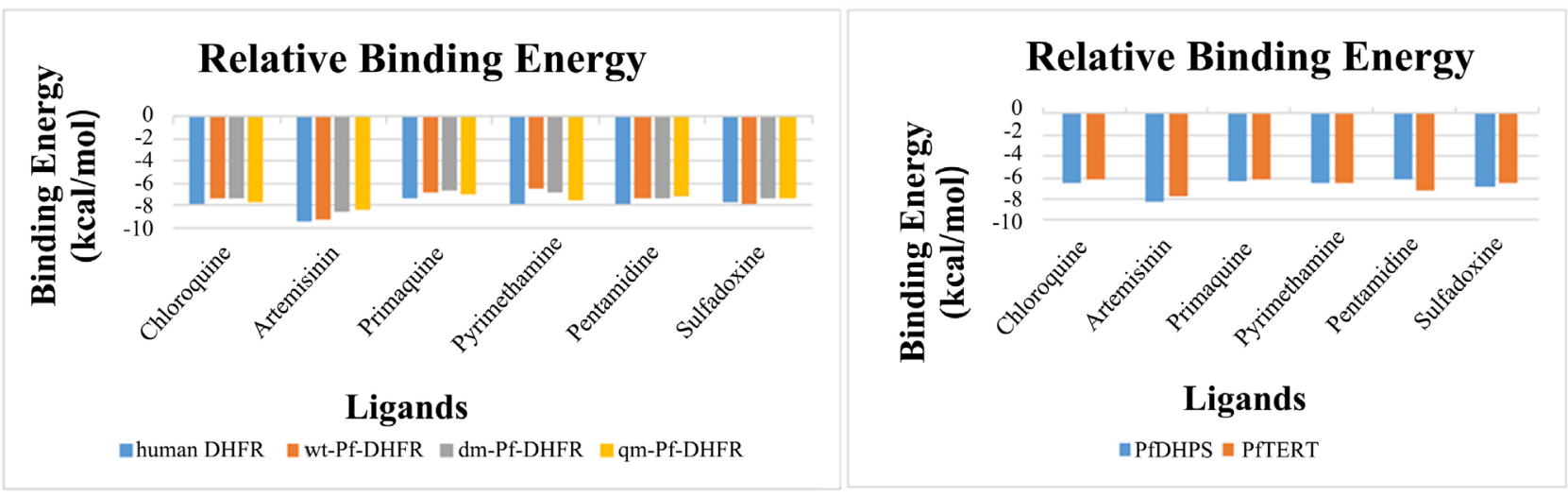

(A)

(B)

Figure 4. Relative binding energies between antimalarial drugs and folate enzymes. (A) DHFR receptors and (B) $p f-T E R T$ and $p f-D H P S$. 
homology modeling (Figure 4(B)). The strength of these interactions does vary from receptor to receptor and depended on ligands applied. Specifically the affinity of the ligands with folate pathway enzyme DHFR-TS dimer is stronger compared to $p f-D H P S$ and $p f-T E R T$. The benchmark drug used to treat malaria formed the most stable complexes with all the receptors. The affinity of all the ligands with folate pathway enzyme DHFR is more favorable compared to DHPS and $p f-T E R T$. For example, the relative binding energy between the antimalarial drugs and receptor were: -7.0 to $-10.0 \mathrm{kcal} / \mathrm{mol}$, for DHFR-TS, -6.00 to -7.00 $\mathrm{kcal} / \mathrm{mol}$ for $p f-T E R T$, and -6.00 to $-9.00 \mathrm{kcal} / \mathrm{mol}$ for $p f-D H P S$.

\subsection{Binding Site Residues and Complexes}

In this section, we explore the residues and binding pockets in the receptors to identify key features that favor binding. The binding pockets of the receptors are populated by polar and nonpolar residues positioned to form hydrogen bonds, electrostatic, Van Der Waals and hydrophobic interactions with the ligands (Figure 5). The combination of residues, their orientation in the active site of $p f$-DHFR, $p f-D H P S$ and $p f-T E R T$ receptors is critical for variation in affinity (Figure 5 and Figure 6). The binding residues for rest of complexes are summarized in supplemental materials (Table SM2, Figure SM1, Figures SM7-SM12).

The complex between h-DHFR and pyrimethamine, is stabilized by hydrophobic interactions involving residues F31, F34, I60, L67 and L22 (Figure 5). The $w t$-pf-DHFR-pyrimethamine complex is stabilized by hydrogen bonding involving Y170 and I164, Van Der Waals interactions involving I112, F58, L119, L46 and G41 (Figure SM2). The $d m$-pf-DHFR-pyrimethamine, complex is stabilized by hydrogen bonding with S111 and G44, hydrophobic interactions with F58, L40 and L46 (Figure SM3). The qm-pf-DHFR-pyrimethamine complex, is stabilized by three hydrogen bonding interactions involving L40, A16, N54 and hydrophobic interactions with V195, L164 and L46 (Figure SM4). The increase in hydrogen bonding contacts in the interactions between the mutant forms of DHFR and pyrimethamine is an important finding which may indicate some unique features of pyrimethamine. The pyrimethamine complexes formed are stabilized by and hydrophobic interactions involving non-polar residues. The binding site residues for $p f$-DHFR-TS receptor include: Isoleucine 14(I14), Leucine 46(L46), Phenylalanine 58(F58), Serine 111(S111), Isoleucine 164(I164), Threonine 185(T185), Phenylalanine 58(F58) is identified as a key residue involved in affinity with Plasmodium enzyme receptors. This residue has been identified as a key factor in the affinity of novel antimalarial ligands like P218 with DHFR [21]. Our calculation also identifies F58 presence in the wt-pf-DHFRpyrimethamine complex and $d m$-pf-DHFR-pyrimethamine complex (Table SM2) as critical for complex formation.

The active site of $p f$-DHFR, $p f-D H P S$ and $p f$-TERT receptors contains a mixture of polar and non-polar residues. The difference in affinity observed between these receptors towards the antimalarial ligands could be explained by the size 


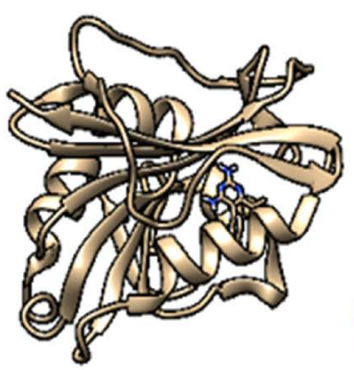

(A)

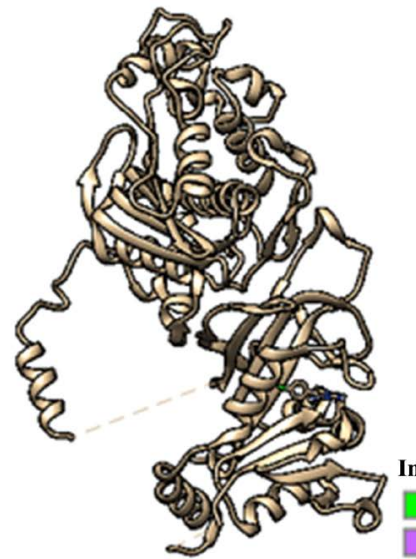

(C)

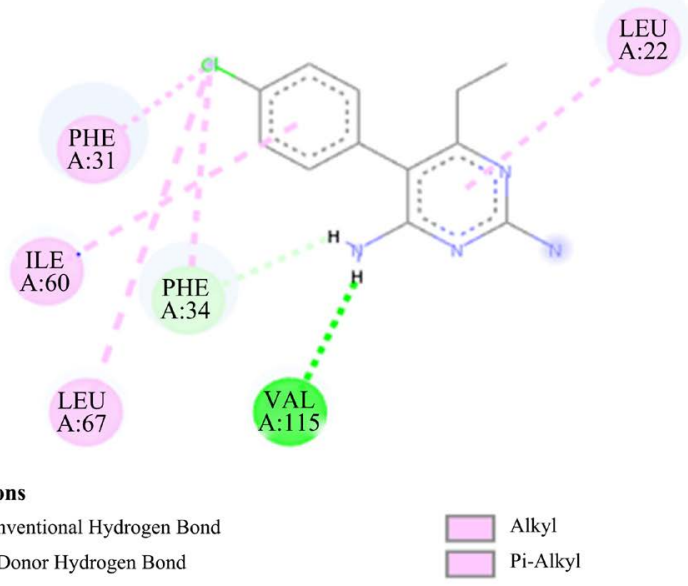

(B)

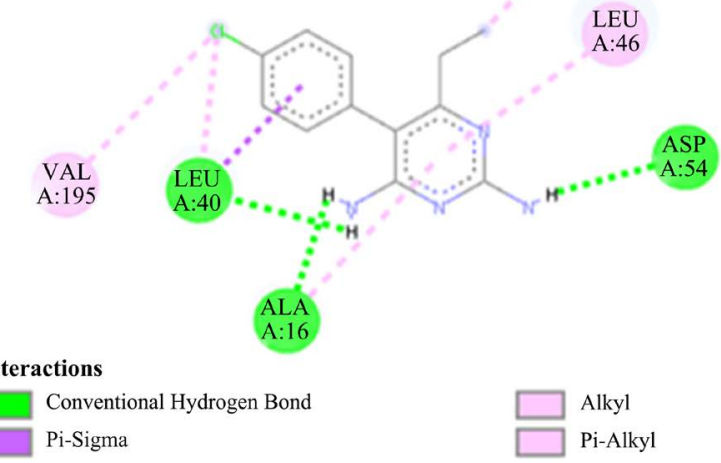

(D)

Figure 5. (A) h-DHFR-pyrimethamine complex and (B) 2D interaction diagram; (C) $q m$ - $p f$-DHFR-pyrimethamine complex and (D) 2D interaction diagram.

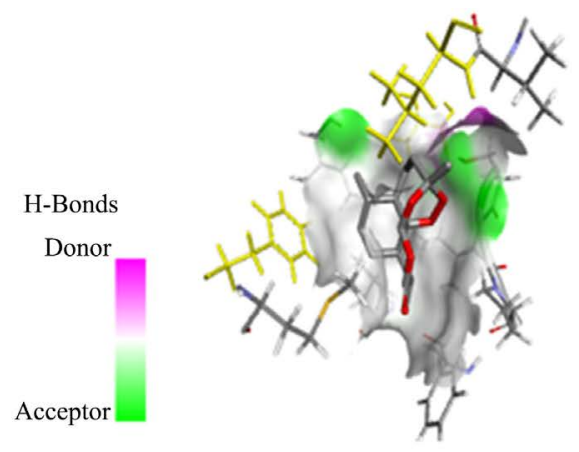

(A)

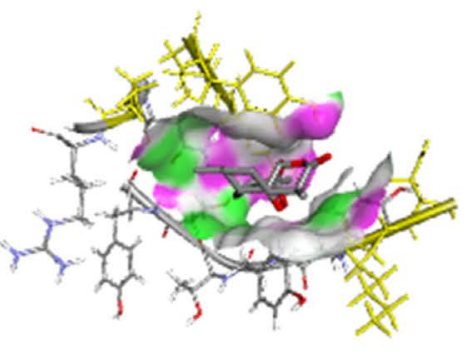

(B)

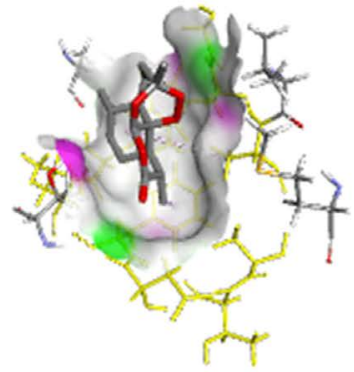

(C)

Figure 6. Surface created by amino acid residues around ligands. (A) $p f$-DHFR-TS-artemisinin complex; (B) $p f$-DHPS-artemisinin complex; (C) pf-TERT-artemisinin complex.

and nature (polar or nonpolar, acid or basic) of amino acids residues around the ligand (Figure 6). The binding pocket of the $p f$-DHFR-TS-artemisinin complex (Figure SM5), comprise multiple hydrophobic residues, involved in strong Van 
Der Waals interactions with the cycloalkane rings or artemisinin. The snapshot of the molecular surface of the artemisinin described in (Figure 6(A)) also points to a strong contribution from non-polar interactions. The polar S111 and T108 are responsible for the strong polar contribution to the stability of the complex (Figure 6). The binding pocket of the $p f$-DHPS-artemisinin complex comprises multiple basic amino acids (Arg and Lys), acidic amino acids (Asp, Asn) and polar amino acids (Ser, Thr, Tyr, Asn, and Asp). The reduced affinity for interactions here could be a result of the diminished impact of Van Der Waals interaction with the large hydrophobic cyclic rings of artemisinin. The energy obtained could be explained by the strong presence of polar amino acids. The favorable energy and stability of the complex involving $p f$-TERT-artemisinin (Figure SM6) is due to both polar and non-polar contacts as we an abundance of nonpolar amino acids mixed with polar ones like D427 and T481.

\section{Conclusion}

In this study, the $3 \mathrm{D}$ structures of $p f-T E R T$ and $p f-D H P S$ enzymes were obtained through homology modelling using chimera and modeller software. The interactions of 6 antimalarial drugs with $p f$-TERT, $p f$-DHPS models and DHFR enzymes were screened using molecular docking with AutoDock Vina. The docking calculations show that all the ligands involved formed stable complexes with the receptors. DHFR receptors, however, seem to have a high affinity compared to $p f-T E R T$ and $p f-D H P S$. Their simulations show that the benchmark drug artemisinin shows the strongest affinity with all the receptors and this can be attributed to the size, shape and bipolar nature of the ligand. This bipolar nature ensures one side is mostly hydrophobic and the other polar. We observe that the stable complexes are formed when the ligands successfully bind using both favorable polar and non-polar contacts. The performed study provides us with a pharmacophore that can be used for the development of new antimalarial chemotherapies.

\section{Funding Details}

This work was supported with funds from the school of science and technology, Georgia Gwinnett College.

\section{Acknowledgements}

Djogang thanks the School of Science and Technology, Chemistry, Georgia Gwinnett College (USA) for providing the software used in this work.

\section{Conflicts of Interest}

The authors declare no conflicts of interest for this research work.

\section{References}

[1] Klonis, N., Creek, D.J. and Tilley, L. (2013) Iron and Heme Metabolism in Plasmo- 
dium falciparum and the Mechanism of Action of Artemisinins. Current Opinion in Microbiology, 16, 722-727. https://doi.org/10.1016/j.mib.2013.07.005

[2] World Health Organisation: World Malaria Report 2012.

[3] Zhang, K. and Rathod, P.K. (2002) Divergent Regulation of Dihydrofolate Reductase between Malaria Parasite and Human Host. Science, 296, 545. https://doi.org/10.1126/science.1068274

[4] Hyde, J.E. (2005) Exploring the Folate Pathway in Plasmodium falciparum. Acta Tropica, 94, 191-206. https://doi.org/10.1016/j.actatropica.2005.04.002

[5] Mukinay, C.D., Babalola, R., Sloop, J.C. and Forlemu, N. (2017) Targeting the Plasmodium falciparum Folate Pathway: Molecular Modeling of the Affinity of Sulfonamide Derivatives and Isoforms of Dihydrofolate Reductase Thymidylate Synthase. Journal of Undergraduate Chemistry Research, 16, 68-71.

[6] Asrar, A., Neyaz, M.K. and Ikramul, S.H. (2014) Exploiting Unique Structural and Functional Properties of Malarial Glycolytic Enzymes for Antimalarial Drug Development. Malaria Research and Treatment, 2014, Article ID: 451065. https://doi.org/10.1155/2014/451065

[7] Askari, B.S. and Krajinovic, M. (2010) Dihydrofolate Reductase Gene Variation in Susceptibility to Disease and Treatment Outcomes. Current Genomics, 11, 578-583. https://doi.org/10.2174/138920210793360925

[8] Raimondi, M.V., Randazzo, O., La Franca, M., Barone, G., Virgini, E., Rossi, D. and Collina, S. (2019) DHFR Inhibitors: Reading the Past for Discovering Novel Anticancer Agents. Molecules, 24, 1141-1159.

https://doi.org/10.3390/molecules24061140

[9] Ojurongbe, O., Tijani, B.D., Fawole, A.A., Adeyeba, O.A. and Kun, J.F. (2011) Prevalence of Dihydrofolate reductase Gene Mutations in Plasmodium falciparum Isolate from Pregnant Women in Nigeria. Infectious Disease Reports, 3, 1-16.

https://doi.org/10.4081/idr.2011.e16

[10] Parida, P., Kalita, S., Shankar, B., Yadav, R.N.S. and Das, A. (2014) Homology Modeling and Docking Studies of Plasmodium falciparum Telomerase Reverse Transcriptase with Berberine and Some of Its Derivatives. Bangladesh Journal of Pharmacological, 9, 96-104. https://doi.org/10.3329/bjp.v9i1.17535

[11] Burley, S.K., Berman, H.M., Kleywegt, G.J., Markley, J.L., Nakamura, H. and Velankar, S. (2017) Protein Data Bank (PDB): The Single Global Macromolecular Structure Archive. In: Protein Crystallography, Springer, Berlin, 627-641. https://doi.org/10.1007/978-1-4939-7000-1_26

[12] Azam, S. and Abbasi, S.W. (2013) Molecular Docking Studies for the Identification of Novel Melatoninergic Inhibitors for Acetylserotonin-O-methyltransferase Using Different Docking Routines. Theoretical Biology and Medical Modelling, 10, 63-80. https://doi.org/10.1186/1742-4682-10-63

[13] Wishart, D.S., Feunang, Y.D., Guo, A.C., Lo, E.J., Jason, A.M., Grant, R., Sajed, T., Johnson, D., Li, C., Sayeeda, Z., Assempour, N., Iynkkaran, I., Liu, Y., Maciejewski, A., Gale, N., Wilson, A., Chin, L., Cummings, R., Le, D., Pon, A., Knox, C. and Wilson, M. (2017) DrugBank 5.0: A Major Update to the DrugBank Database for 2018. Nucleic Acids Research, 46, D1074-D1082. https://doi.org/10.1093/nar/gkx1037

[14] Gromiha, M.M., Nagarajan, R. and Selvaraj, S. (2018) Protein Structural Bioinformatics: An Overview. In: Reference Module in Life Sciences, Elsevier, Amsterdam, 1-14. https://doi.org/10.1142/9789811211874_0007

[15] Skariyachan, S. and Garka, S. (2018) Exploring the Binding Potential of Carbon 
Nanotubes and Fullerene Towards Major Drug Targets of Multidrug Resistant Bacterial Pathogens and Their Utility as Novel Therapeutic Agents. In: Fullerens, Graphenes and Nanotubes, Elsevier, Amsterdam, 1-29.

https://doi.org/10.1016/B978-0-12-813691-1.00001-4

[16] Wu, C.H. (2006) The Universal Protein Resource (UniProt): An Expanding Universe of Protein Information. Nucleic Acids Research, 34, 187-191.

https://doi.org/10.1093/nar/gkj161

[17] Chen, J.E., Huang, C.C. and Ferrin, T.E. (2014) RRDistMaps: A UCSF Chimera Tool for Viewing and Comparing Protein Distance Maps. Bioinformatics, 31, 1484-1486. https://doi.org/10.1093/bioinformatics/btu841

[18] Fiser, A. and Sali, A. (2003) Modeller: Generation and Refinemet of Homology-Based Protein Structure Models. Macromolecular Crystallography, 374, 461-491. https://doi.org/10.1016/S0076-6879(03)74020-8

[19] Pourseif, M.M., Yousefpour, M., Aminianfar, M., Moghaddam, G. and Nematollahi, A. (2019) A Multi-Method and Structure-Based in Silico Vaccine Designing against Echinococcus granulosus Through Investigating Enolase Protein. Bioimpacts, 9, 131-144. https://doi.org/10.15171/bi.2019.18

[20] Gangjee, A., Li, W., Kisliuk, R.L., Cody, V., Pace, J., Piraino, J. and Makin, J. (2009) Design, Synthesis, and X-Ray Crystal Structure of Classical and Nonclassical 2-Amino-4-oxo-5-substituted-6-ethylthieno[2,3-d]pyrimidines as Dual Thymidylate Synthase and Dihydrofolate Reductase Inhibitors and as Potential Antitumor Agents. Journal of Medicinal Chemistry, 52, 4892-4902. https://doi.org/10.1021/jm900490a

[21] Yuthavong, Y., Tarnchompoo, B., Vilaivan, T., Chitnumsub, P., Kamchonwongpaisan, S., Charman, A.S. and Matthews, D. (2012) Malaria Dihydrofolate Reductase as a Paradigm for Drug Development against a Resistance-Compromised Target. Proceeding of the National Academy of Sciences, 109, 16823-16828.

https://doi.org/10.1073/pnas.1204556109

[22] Vanichtanankul, J., Taweechai, S., Uttamapinant, C., Chitnumsub, P., Vilaivan, T., Yuthavong, Y. and Kamchonwongpaisana, S. (2012) Combined Spatial Limitation around Residues 16 and 108 of Plasmodium falciparum Dihydrofolate Reductase Explains Resistance to Cycloguanil. Antimicrobial Agents and Chemotherapy, 56, 3928. https://doi.org/10.1128/AAC.00301-12

[23] Trott, O. and Olson, A.J. (2010) AutoDock Vina: Improving the Speed and Accuracy of Docking with a New Scoring Function, Efficient Optimization, and Multithreading. Journal of Computational Chemistry, 31, 455-461.

https://doi.org/10.1002/jcc.21334

[24] Fuhrmann, J., Rurainski, A., Lenhof, H.P. and Neumann, D. (2010) A New Lamarckian Genetic Algorithm for Flexible Ligand-Receptor Docking. Journal of Computational Chemistry, 31, 1911-1918. https://doi.org/10.1002/jcc.21478

[25] Scherf, A. (2001) Plasmodium Telomeres: A Pathogen's Perspective. Current Opinion in Microbiology, 4, 409-414. https://doi.org/10.1016/S1369-5274(00)00227-7

[26] Osamor, V.C. (2010) The Etiology of Malaria Scourge: A Comparative Study of Endemic Nations of Africa and Asia. Journal of Biological Sciences, 10, 440-447. https://doi.org/10.3923/jbs.2010.440.447 


\section{Abbreviations}

DHFR: dihydrofolate reductase.

DHPS: dihydropteroate synthetase.

pf. Plasmodium falciparum.

pf-TERT: Plasmodium telomerase reverse-transcriptase.

\section{Appendix A. Supplementary Data}

Supplementary data to this article can be found online at https://doi.org/..............

\section{Supplemental Material}

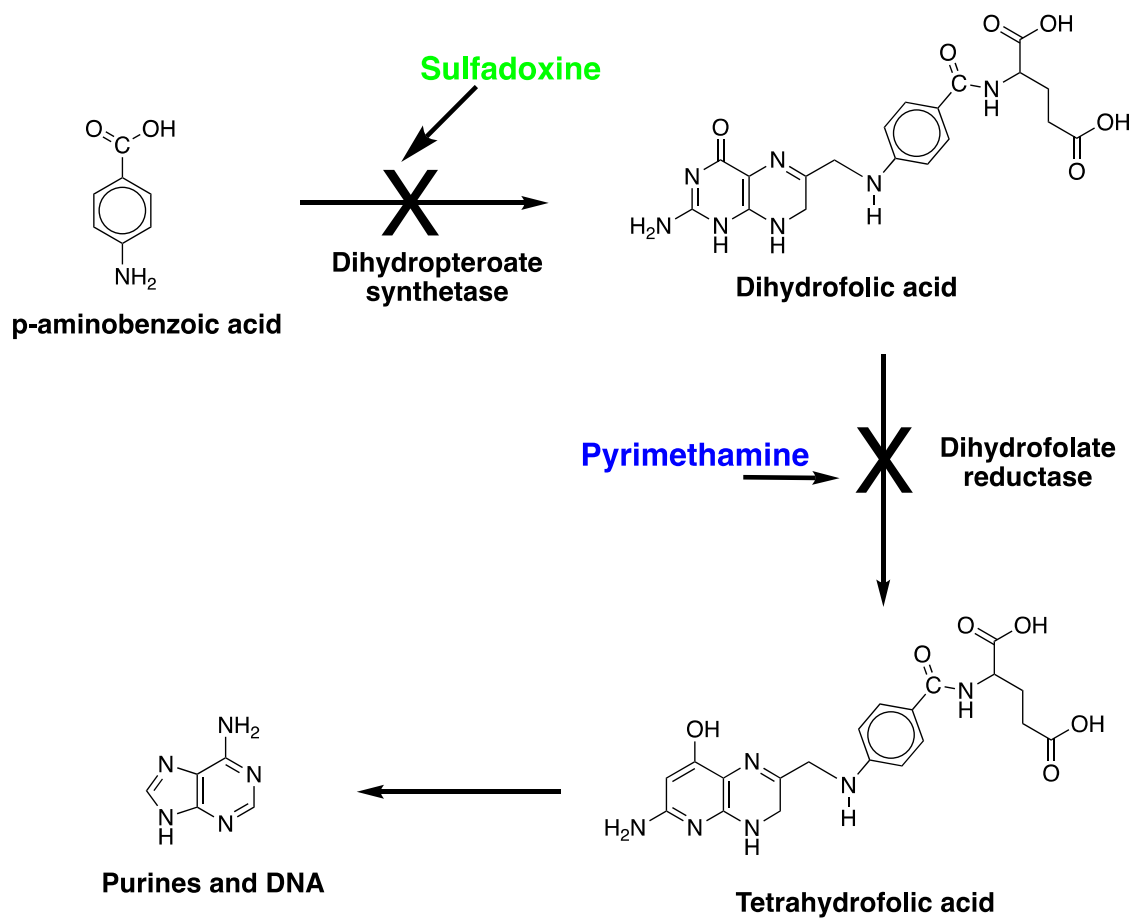

Scheme 1. Folate pathway and antifolate drug against malarial.

Table SM1. Sequence similarity scores between target protein and identified template structures.

\begin{tabular}{cccc}
\hline Target Sequence & Template ID & Sequence Identity & Scores \\
\hline \multirow{2}{*}{$p f-T E R T$} & 1. 1ONF_A & 99.60 & 1008 \\
& 2.5GRT_A & 44.10 & 352 \\
& 3. 3DK9_A & 44.54 & 340 \\
pf-DHPS & 1.6KCM_A & 99.0 & 410 \\
& 2. 6KCL_A & 99.0 & 412 \\
& 3.6JWT_A & 99.0 & 411 \\
\hline
\end{tabular}


Table SM2. Residues involved different complexes and their energies

\begin{tabular}{ccccccc}
\hline Residue Type & $\begin{array}{c}\text { h-DHFR } \\
\text { Pyrimethamine }\end{array}$ & $\begin{array}{c}\text { wt-DHFR } \\
\text { Pyrimethamine }\end{array}$ & $\begin{array}{c}d m \text {-DHFR } \\
\text { Pyrimethamine }\end{array}$ & $\begin{array}{c}q m \text {-DHFR } \\
\text { Pyrimethamine }\end{array}$ & $\begin{array}{c}p f \text {-DHPS } \\
\text { Pyrimethamine }\end{array}$ & $\begin{array}{c}p f-T E R T \\
\text { Pyrimethamine }\end{array}$ \\
\hline $\begin{array}{c}\text { Hydrophobic } \\
\text { Hydrogen Bonding }\end{array}$ & F31, I60, F34, L67, L22 & I112, F58, L119, L46 & F58, L40, L46 & V195, L164, L46 & R28 & V351, L353, K214 \\
Energy Kcal/mol & V115 & Y170, I164 & S111, G44 & L40, A16, N54 & I79, N25, Y159, S161 & T393, I381, G395 \\
\hline & Artemisinin & Artemisinin & Artemisinin & Artemisinin & Artemisinin & Artemisinin \\
\hline Hydrophobic & F34 & F58, L46 & L46 & L46 & Y80 & A468, F465 \\
Hydrogen Bonding & S59 & S108 & I112 & $/$ & Y80 & / \\
Energy Kcal/mol & -9.4 & -9.2 & -8.5 & -8.4 & -8.3 & -7.7 \\
\hline
\end{tabular}

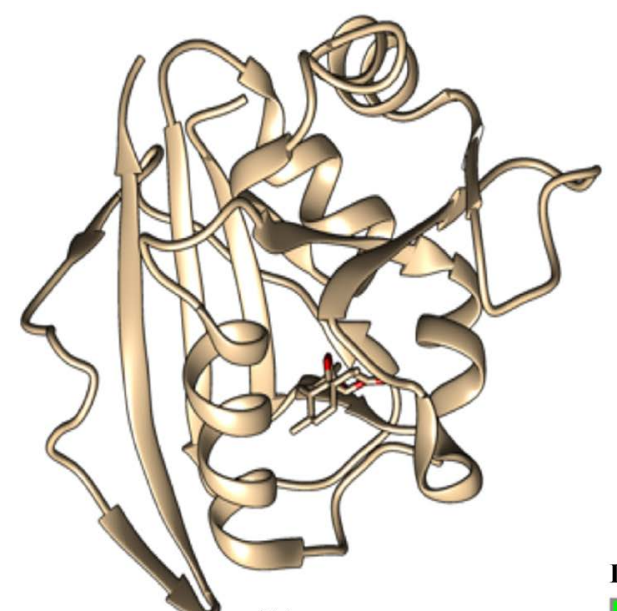

(A)

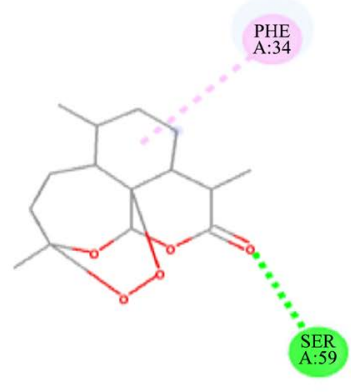

(B)

Interactions

Conventional Hydrogen Bond $\square$ Pi-Alkyl

Figure SM1. (A) h-DHFR-artemisinin complex; (B) 2D interaction diagram.

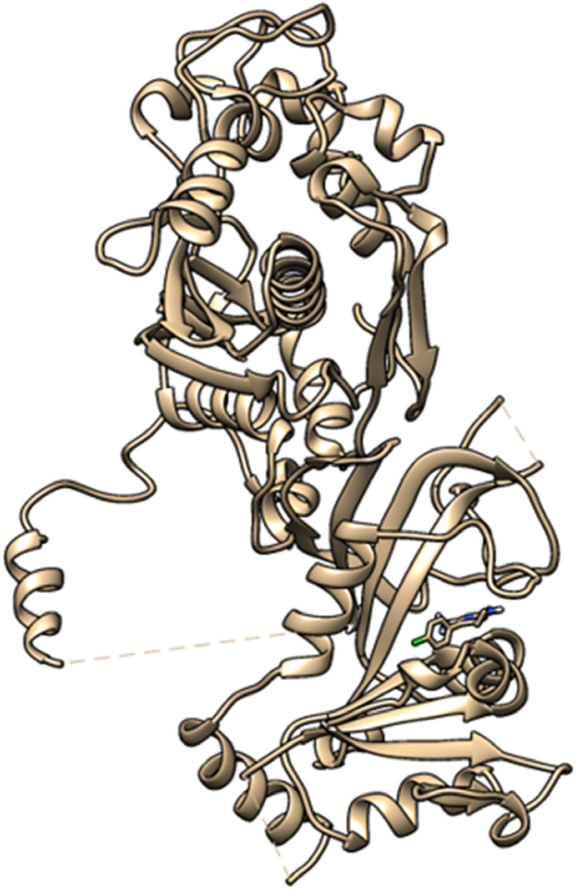

(A)

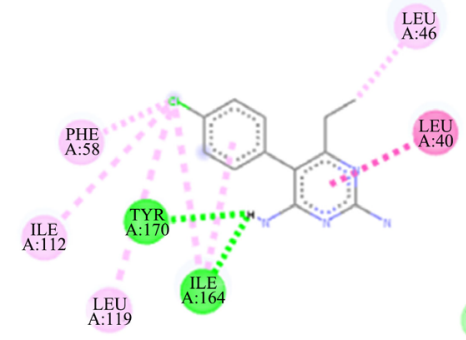

(B)

Figure SM2. (A) wt-pf-DHFR-pyrimethamine complex; (B) 2D interaction diagram. 


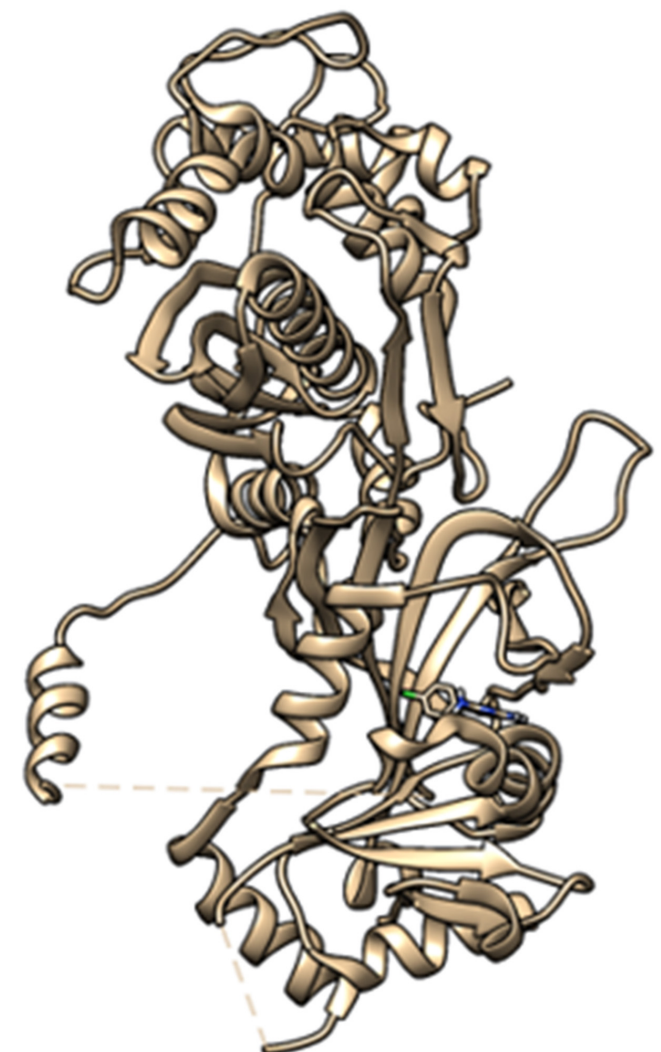

(A)

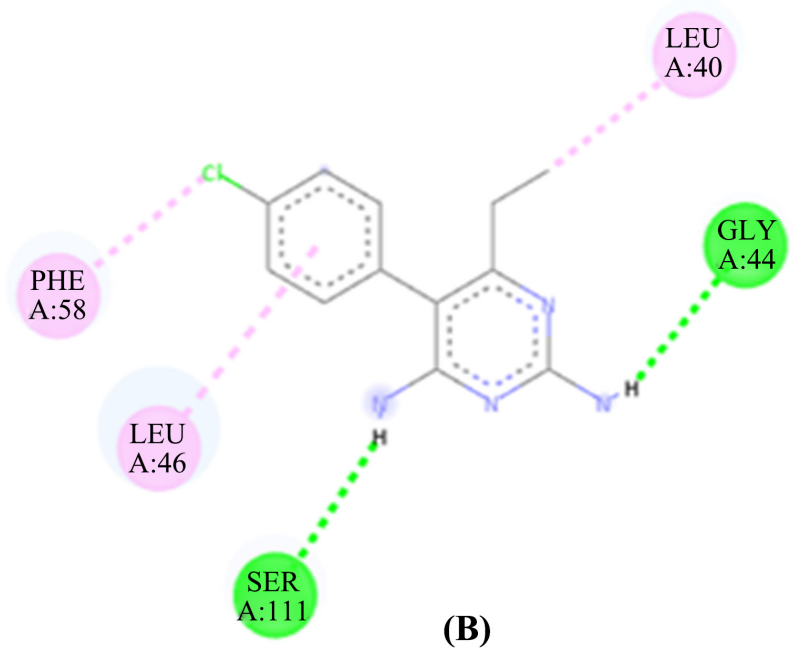

Interactions

Conventional Hydrogen Bond Akyl

Figure SM3. (A) $d m$-pf-DHFR-pyrimethamine complex; (B) 2D interaction diagram.

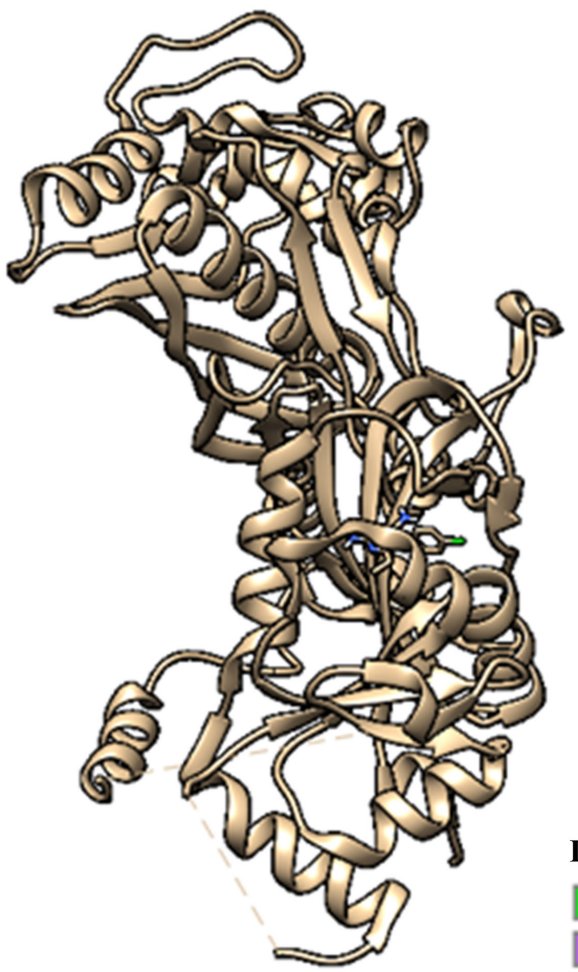

(A)

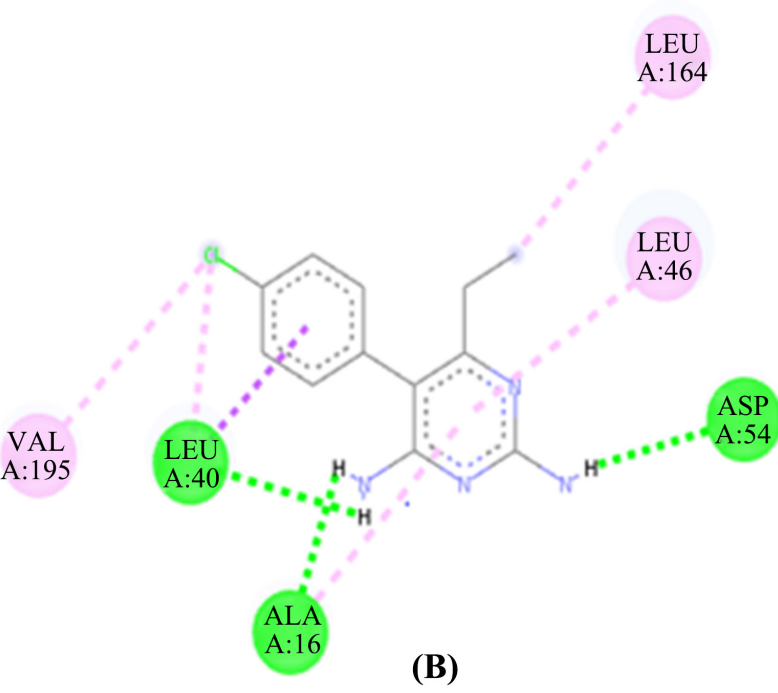

Interactions

Conventional Hydrogen Bond Pi-Sigma

Figure SM4. (A) $q m-p f$-DHFR-pyrimethamine complex; (B) 2D interaction diagram. 


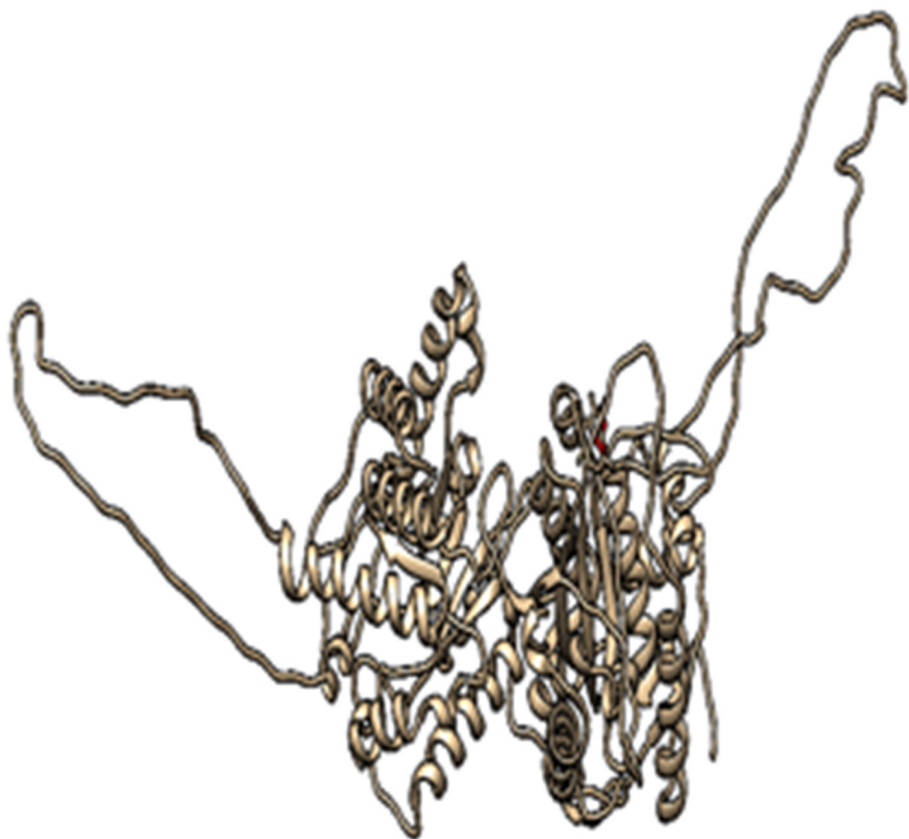

(A)

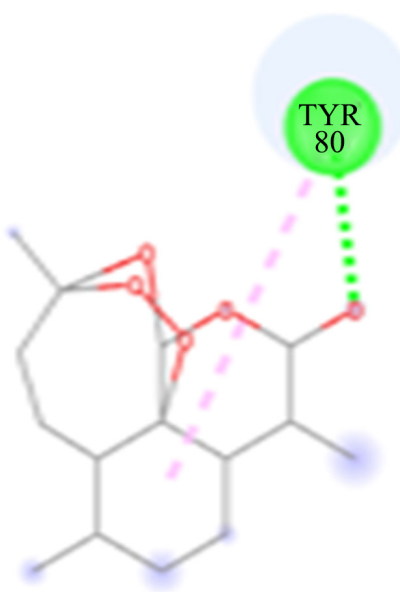

(B)

\section{Interactions}

Conventional Hydrogen Bond
Pi-Alkyl

Figure SM5. (A) pf-DHPS-artemisinin complex; (B) 2D interaction diagram.

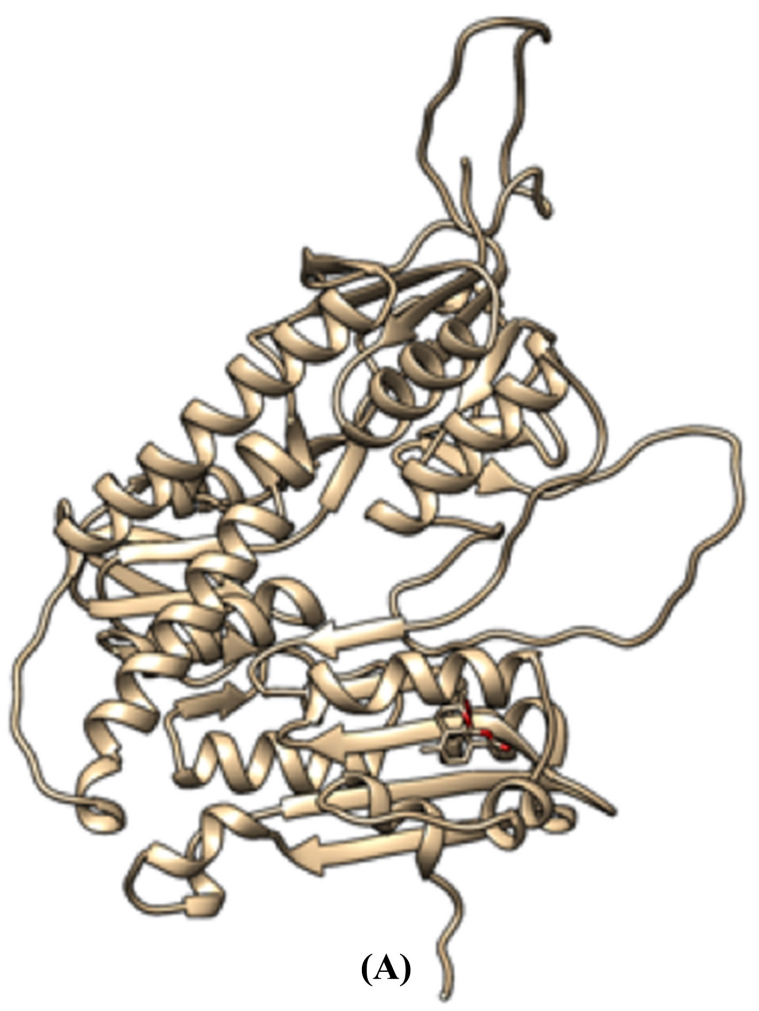

Figure SM6. (A) $p f-T E R T$-artemisinin complex; (B) 2D interaction diagram.

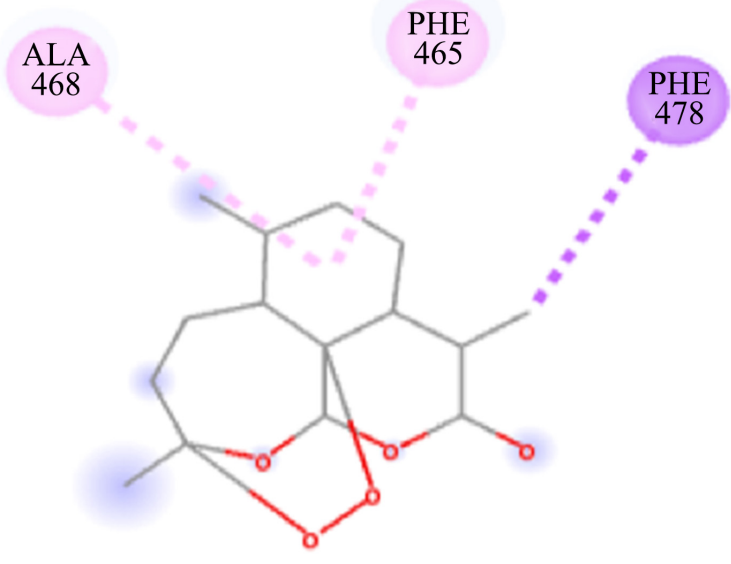

(B)

\section{Interactions}

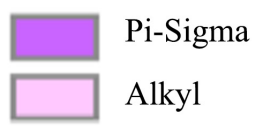

Alkyl
Pi-Alkyl 


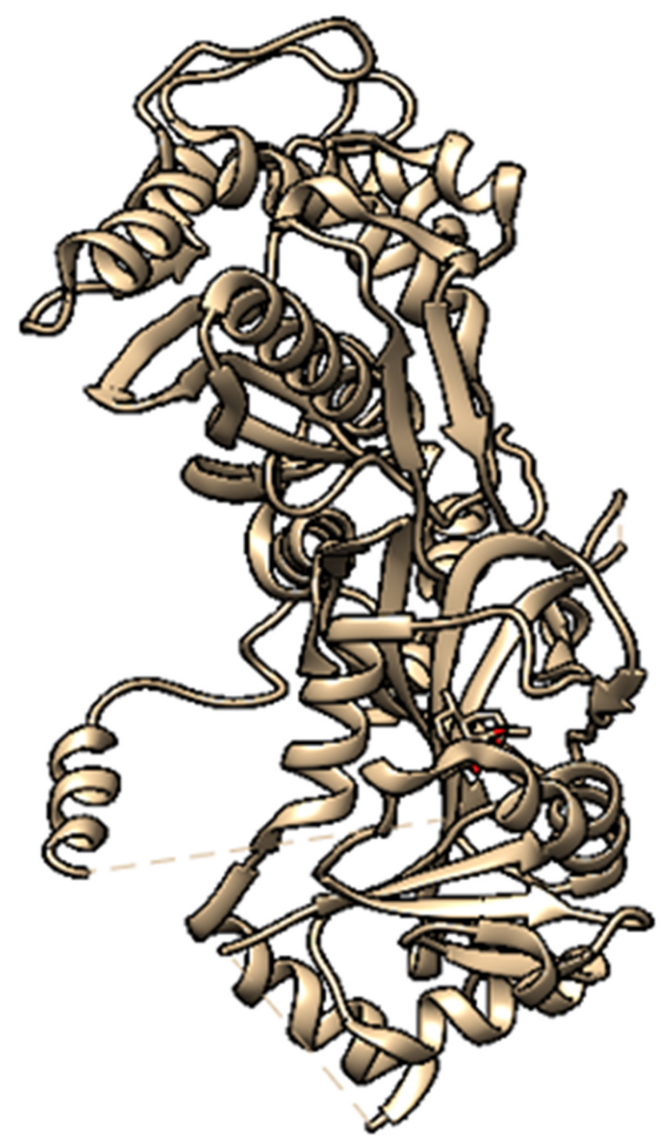

(A)

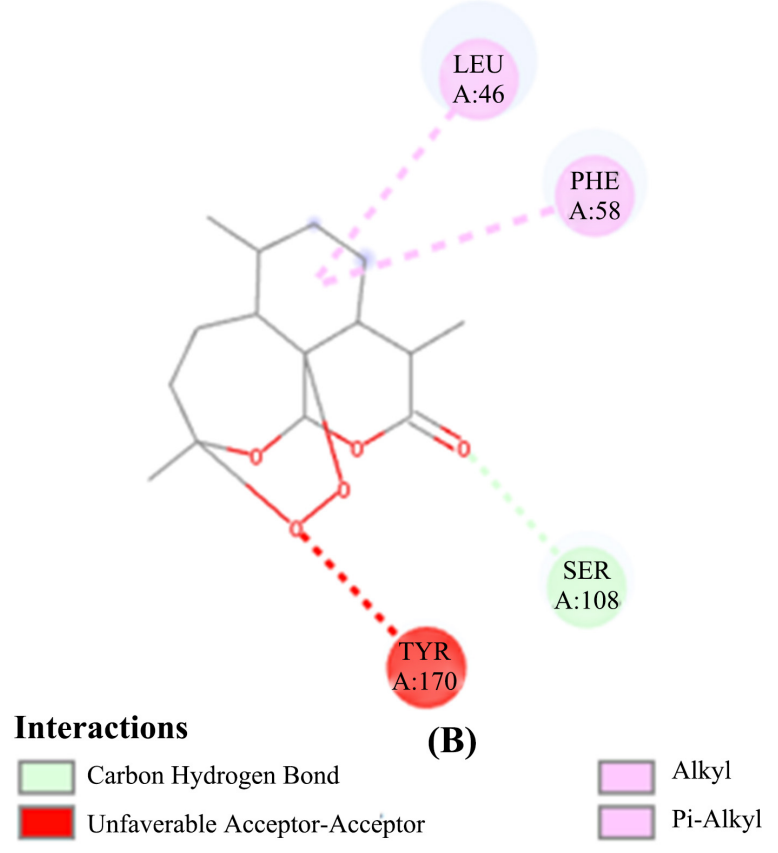

Figure SM7. (A) $w t-p f$-DHFR-artemisinin complex; (B) 2D interaction diagram.

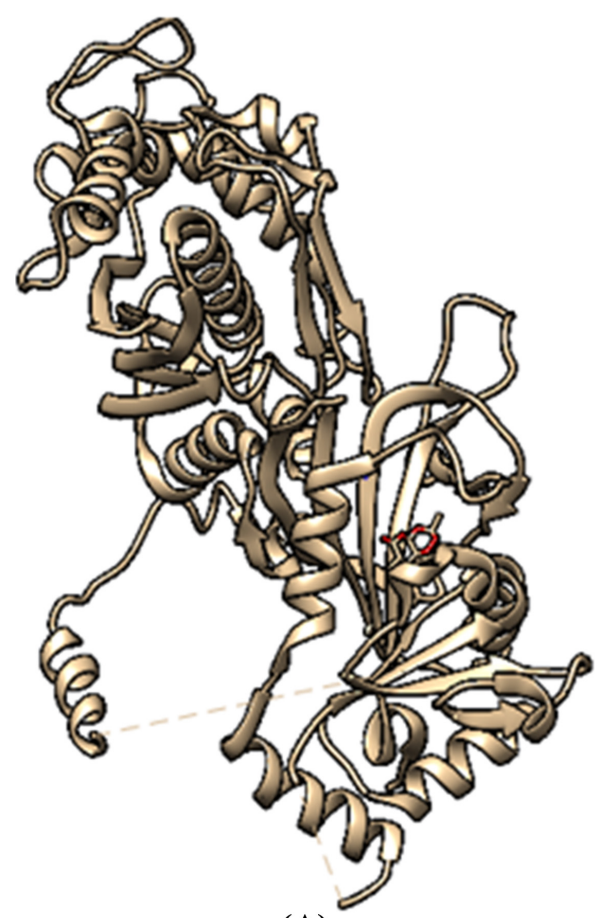

(A)

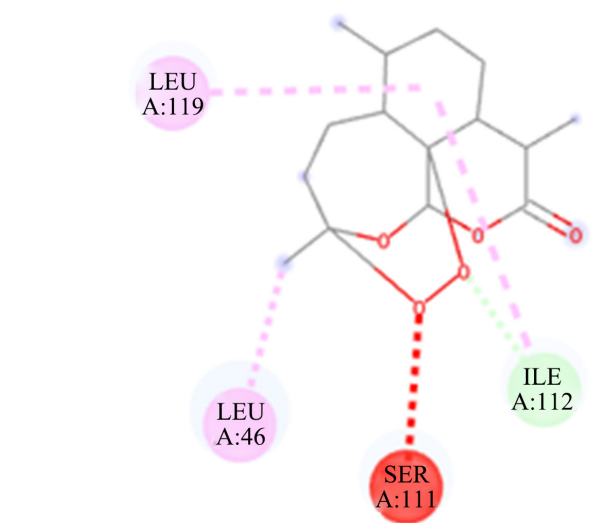

(B)

Figure SM8. (A) $d m$-pf-DHFR-artemisinin complex; (B) 2D interaction diagram. 

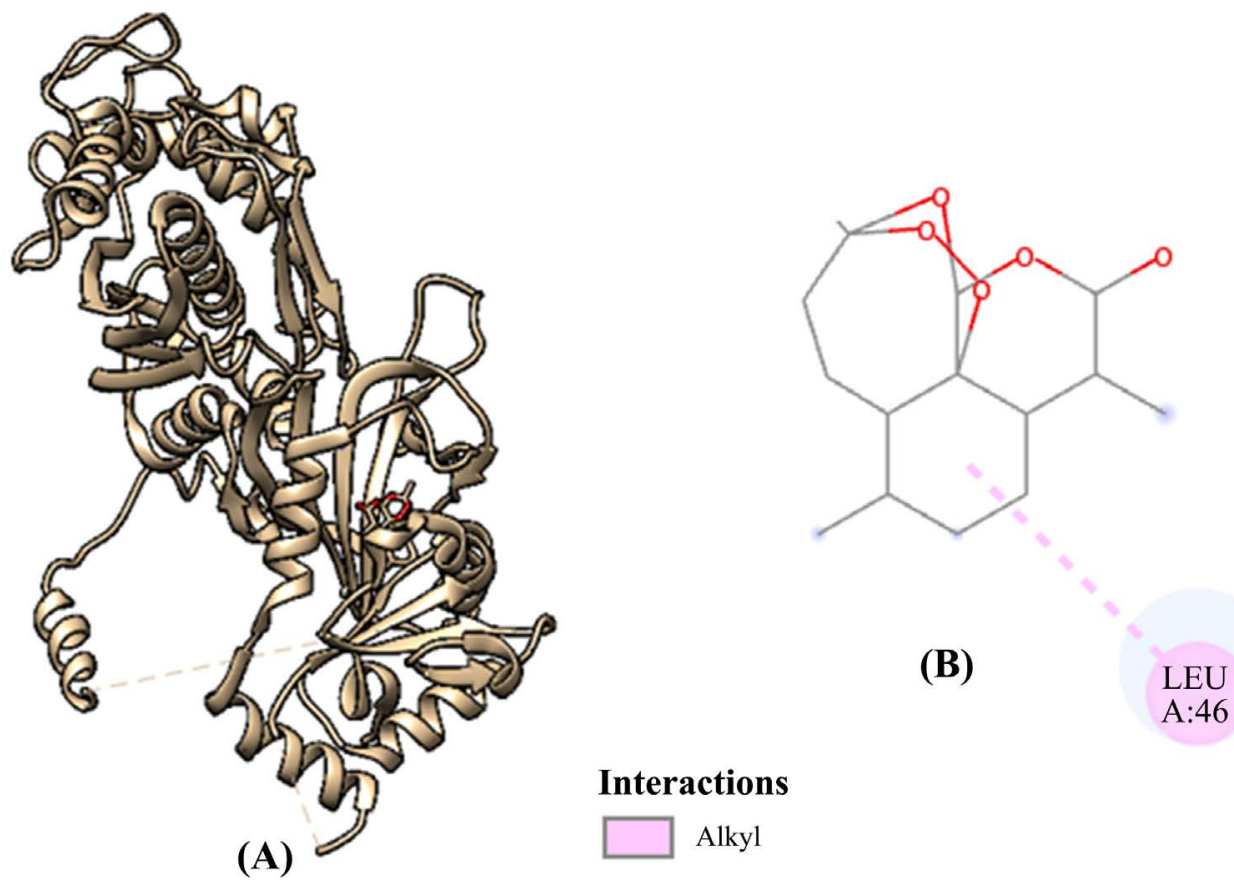

\section{Interactions}

Figure SM9. (A) $q m-p f$-DHFR-artemisinin complex; (B) 2D interaction diagram.

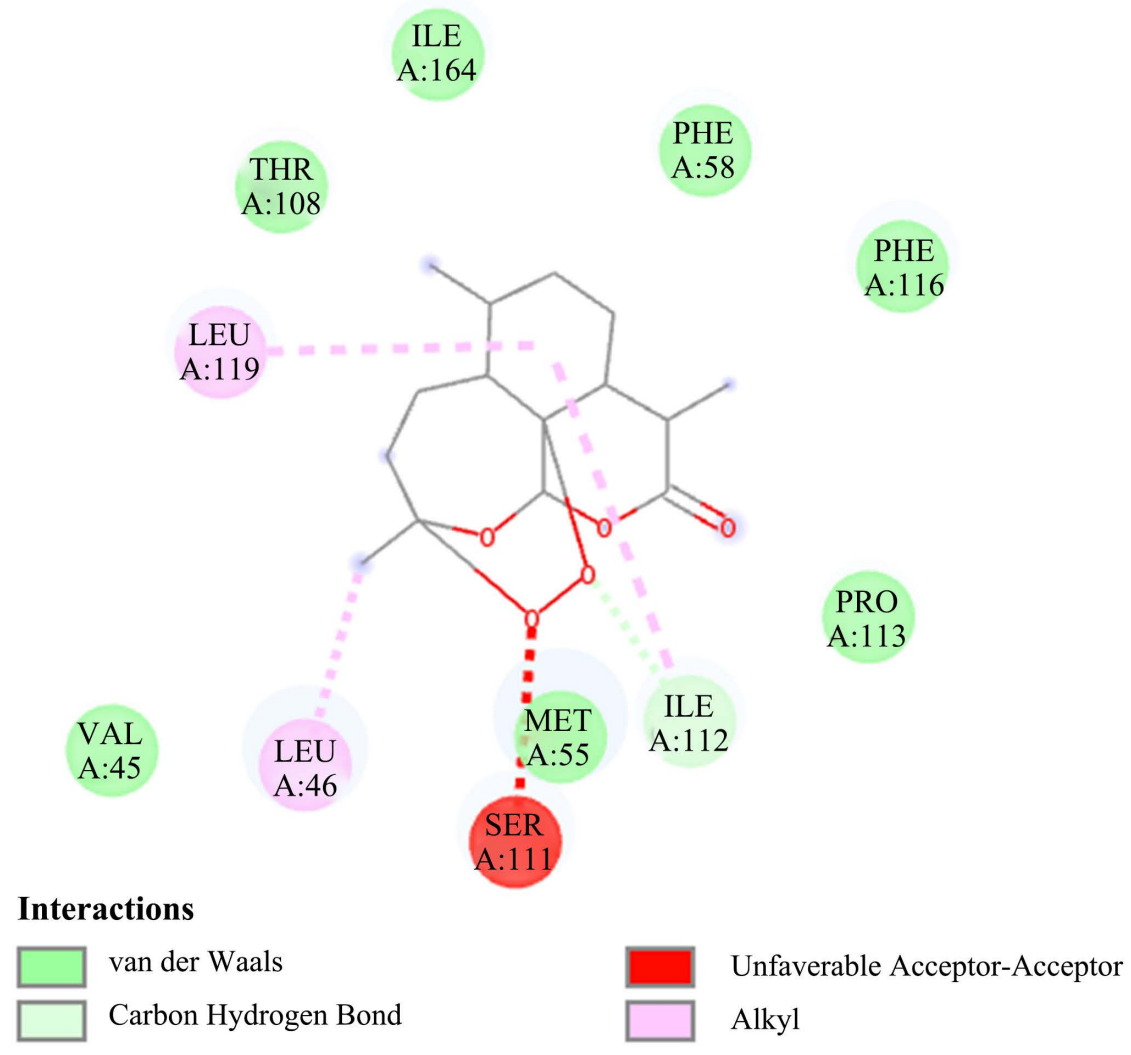

Figure SM10. Binding domain amino acids residues involved $p f$-DHFR-TS-artemisinin complex. 


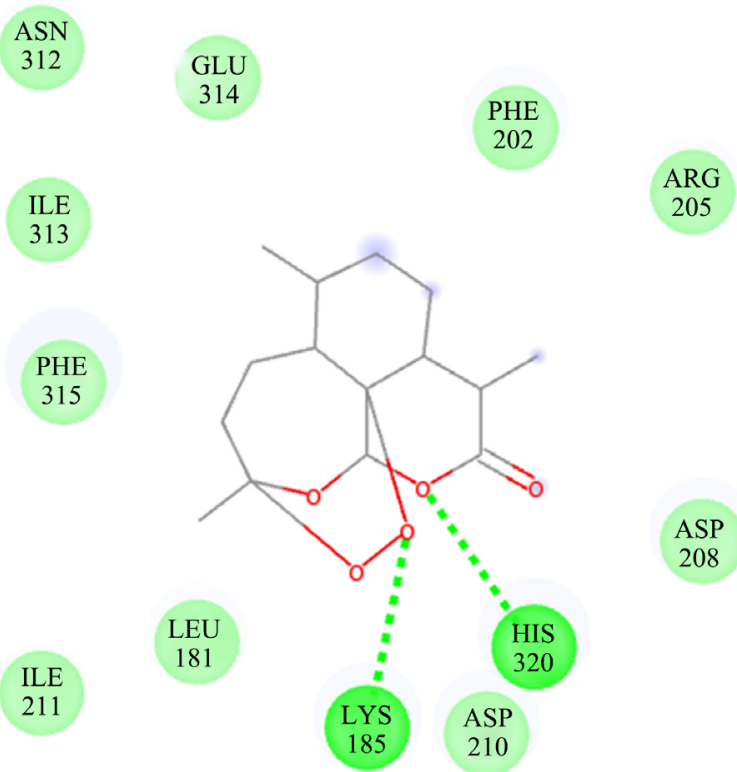

\section{Interactions}

van der Waals

Figure SM11. Binding domain amino acids residues involved $p f$-DHPS-artemisinin complex.
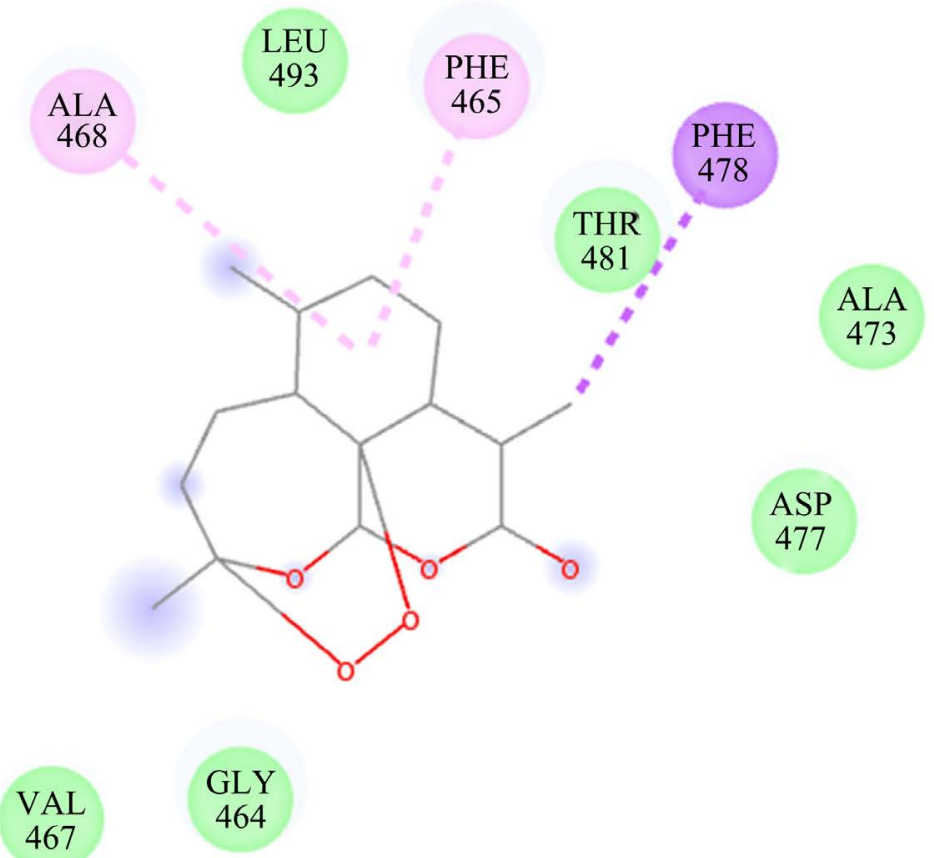

\section{Interactions}

$\square$ van der Waals
Pi-Sigma

Figure SM12. Binding domain amino acids residues involved $p f-T E R T$-artemisinin complex. 